\title{
Mathematical Principles and Optimal Design Solutions to Compensation for the Pendulum Temperature Dilatation
}

\author{
Branislav Popkonstantinović ${ }^{1}$, Ljubomir Miladinović ${ }^{2}$, Marija \\ Obradovic $\mathbf{c}^{3}$, Gordana Ostojić ${ }^{4}$, Stevan Stankovski ${ }^{5}$ \\ ${ }^{1}$ Faculty of Mechanical Engineering, Belgrade, Serbia, \\ bariton@afrodita.rcub.bg.ac.rs \\ ${ }^{2}$ Faculty of Mechanical Engineering, Belgrade, Serbia, \\ lmiladinovic@mas.bg.ac.rs \\ ${ }^{3}$ Faculty of Civil Engineering, Belgrade, Serbia, \\ marijaobradovic.masha@gmail.com \\ ${ }^{4}$ Faculty of Technical Sciences, Novi Sad, Serbia, goca@uns.ac.rs \\ ${ }^{5}$ Faculty of Technical Sciences, Novi Sad, Serbia, stevan@uns.ac.rs
}

\begin{abstract}
The paper analyzes the effects of pendulum temperature dilatation on the timepiece running and accounts for the basic mathematical principles that neutralization of these detrimental effects are based upon. Both preliminary and detailed calculations are presented, as well as some design solutions leading to technically acceptable pendulum temperature dilatation. Explanation is given for the design of bimetal gridiron pendulum, wood pendulum with a lead weight, mercury pendulum, and pendulum with an Invar alloy rod.
\end{abstract}

\section{Introduction}

Numerous factors affect the uniform running of a timepiece, however, in both quality and quantity terms the most prominent are those caused by temperature changes. This paper is devoted to the pendulum thermal dilatation, the most detrimental of all detrimental effects, as well as to the explanation of the basic principles that elimination of this effect is based upon. Both preliminary and detailed calculations were performed, resulting in some design solutions to almost perfect compensation for the pendulum temperature dilatation. 
Notwithstanding the fact that the problem of thermal compensation of oscillators, especially of clock pendulums, is old more than 200 years, it still represents the respectable subject of research in modern science and technology. Thus, in [1], Popkonstantinović, B. et al. exposed the analytical method for the pendulum thermal compensation considering not only the mass center but also the pendulum mass moment of inertia of the first and second order. Actually, fast and efficient mathematical method as well as the practical constructive solutions is given by which the technically acceptable thermal compensation of the long period compound pendulum can be obtained. Popkonstantinović, B. et al., in [2], explain the design, solid modelling and motion simulation of the remontoire mechanism only by which the influence of a pendulum thermal compensation for the clock accuracy can be significant and observable. K. V. Kislov investigates, in [3], the influence of temperature noise on the linear dimensions variation of new broadband seismometers, clarifies which elements of the device are the most sensitive to the ambient temperature variations and determines the level of noise generated by all the elements. In [4], Agatsuma K. Et al. achieved for the first time a direct measurement of the thermal fluctuation of a pendulum in an off-resonant region using a laser interferometric gravitational wave detector and disclosed the conclusion that the measured thermal noise level corresponds to a high quality factor on the order of $10^{5}$ of the pendulum. Jie Luo et al. introduced, in [5], the weighting function by considering the nonlinear least-squares fitting method and the correlation method, and developed a method to calculate the influence of thermal noise on the period measurement in a torsion pendulum. Consequently, they obtained a rigorous formula of thermal noise limit and uncertainty estimation for the pendulum period measurement. This result is significant for precise determination of the Newtonian gravitational constant using the time-of-swing method. A finite element modeling can be used in many different applications [6, 7]. A. Cumming et al. discuss, in [8], the finite element modeling and associated analysis of the loss in quasi-monolithic silica fiber suspensions for future advanced gravitational wave detectors. They emphasized that the detector suspension thermal noise will be an important noise source at operating frequencies between approximately 10 and $30 \mathrm{~Hz}$, and results from a combination of thermo elastic damping, surface and bulk losses associated with the suspension fibers. They concluded that its effects can be reduced by minimizing the thermo elastic loss and optimization of pendulum dilution factor via appropriate choice of suspension fiber and attachment geometry. In [9], V. E. Dzhashitov and V. M. Pankratov considered the control possibility of interconnected mechanical and thermal processes in nonlinear perturbed dynamic systems for irregular motions and parametric temperature perturbations. It is shown that the choice of thermal parameters and mechanical subsystems as well as the introduction of mechanical control subsystem proportional to the temperature gradient between its elements into the feedback can provide both regularization of oscillations and control of oscillations at parametric temperature perturbations. Jie Luo and Dian-Hong Wang, in [10], considered the environment temperature variation and 
unhomogenity of background gravitational field and proposed an improved correlation method to determine the variation period of a torsion pendulum with high precision. This analysis is significant for the determination of gravitational constant with the time-of-swing method. Woodward P., in [11] and [12], and Matthys R. J., in [13], analyzed various methods and technical solution for pendulum thermal compensation and proposed important practical advices for the design of high precision pendulum clocks. In [14], Andri M. Gretarsson et al. consider the problem of pendulum thermal noise in advanced large interferometers for detecting gravity waves. Peter R. Saulson, in [15], analyzed thermal noise in mechanical experiments and constructed models for the thermal noise spectra of systems with more than one mode of vibration, and evaluated a model of a specific design of pendulum suspension for the test masses in a gravitational-wave interferometer.

\section{The Pendulum Temperature Dilatation}

Any material, therefore the material that the pendulum is made of, changes its dimensions with a change in temperature. This is the linear temperature dilatation phenomenon described by the formula 1 :

$l=l_{0} \cdot\left(1+a \cdot\left(t-t_{0}\right)\right)=l_{0} \cdot(1+a \cdot \theta)$

In this relation, $a$ is the linear coefficient of temperature (thermal) dilatation, and $l$ and $l_{0}$ are the pendulum rod lengths at temperatures $t$ and $t_{0}$. The values of coefficient $a\left[\mathrm{~K}^{-1}\right]$ that defines the relative change of length per unit of basic length and the level of temperature change are given in Table 1 for some materials mentioned in this paper.

If circular error is neglected, the oscillation period of a physical pendulum can be calculated using well-known formulas 2:

$T \approx 2 \pi \sqrt{\frac{J}{g \cdot S}}=2 \pi \sqrt{\frac{\sum m_{i} r_{i}^{2}}{g \cdot \sum m_{i} r_{i}}}=2 \pi \sqrt{\frac{l_{r}}{g}}$

where $J$ and $S$ are square and static moments of inertia and $g$ is the acceleration of gravity. The formulas indicate clearly that:

$$
\begin{aligned}
& J=\sum m_{i} r_{i}^{2} \\
& S=\sum m_{i} r_{i}
\end{aligned}
$$


$l_{r}=\frac{\sum m_{i} r_{i}^{2}}{\sum m_{i} r_{i}}$

where $m_{i}$ and $r_{i}$ in formulas 3, 4 and 5 are masses and, relative to the suspension point, coordinates of the center of gravity of all the parts of a pendulum, and $l_{r}$ is the so-called reduced length of a physical pendulum. By definition, it represents the length of a mathematical pendulum that has the same period of oscillation as a specified physical pendulum. The static moment $S$ determines the coordinate of the center of gravity, while the square moment $J$ describes the geometric distribution of masses around the suspension point and the center of gravity of the entire pendulum.

Table 1

Linear coefficient of temperature dilatation for some materials

\begin{tabular}{|l||l||l||l|}
\hline MATERIAL & $a 10^{-6}\left[K^{-1}\right]$ & MATERIAL & a $10^{-6}\left[K^{-1}\right]$ \\
\hline \hline Invar alloy & 1,2 & Brass & $18-19$ \\
\hline Wood of silver fir & 4 & Lead & 28 \\
\hline Steel $0.1 \%$ C & 12 & Zinc & 39,7 \\
\hline
\end{tabular}

From the very fact that each line coordinate in the above given formulas is susceptible to temperature dilatations, it follows that the physical pendulum eigenoscillations period changes with temperature change. Illustrative example: a pendulum with a period of $\mathrm{T}=2$ seconds $^{1}$, whose rod is made of simple, lowcarbon structural steel, loses about $0.5 \mathrm{~s}$ a day, with temperature rise of $1{ }^{\circ} \mathrm{C}$. If the temperature rises by $10{ }^{\circ} \mathrm{C}$ a day, the clock having such a pendulum is around $36 \mathrm{~s}$ late for 7 days of permanent running. Under identical temperature conditions, the clock that has a geometrically equivalent pendulum installed, but with a rod of the wood of silver fir, is only $12 \mathrm{~s}$ late for 7 days. The described irregularities in a timepiece running were first noticed by English horologists in the $18^{\text {th }}$ Century, so the earliest attempts to correct the irregularities date back to those days.

\section{Principles of Temperature Dilatation Compensation}

In their simplest form, the principles of compensation for the effects of stochastic temperature changes on the uniform running of a timepiece are based on the choice of the material that has the lowest linear coefficient of temperature dilatation. The above given example indicates clearly that a silver-fir wood rod,

1 In horology such a pendulum is traditionally referred to as one second, because pendulums were formerly indicated according to duration of oscillation semi-period. 
compared to a steel one, is less susceptible to the temperature influence. That is why those rods on old tower clocks and so-called Vienna regulators ${ }^{2}$ were manufactured from wood. Later, in the $19^{\text {th }}$ Century, with the discovery of the Invar alloy ${ }^{3}$, this compensation method was improved, but was never perfect enough. More complex design solutions had to be used for astronomical clocks, chronometers, best quality public clocks and even some wall clocks.

To make compensation for the temperature effects as much complete as possible, a pendulum should be manufactured of at least two different types of material. In 1726, Honorable George Graham ${ }^{4}$ of London was the first to make this idea come true. He constructed the so-called mercury pendulum. Mercury is characterized by a high volumetric temperature expansion coefficient $\left(18 \cdot 10^{-5} \mathrm{~K}^{-1}\right)$ and high density $\left(13.6 \mathrm{~kg} / \mathrm{m}^{3}\right)$, which can be utilized for compensation of the pendulum center of gravity displacement due to its rod thermal dilatation. Fig. 1 shows a typical design solution for the mercury pendulum: a vial of glass or cast iron filled with some amount of mercury. The change in pendulum temperature changes both its rod length and mercury column height in a vial, but in the opposite direction. Detailed calculations can be used to determine the needed mercury column height to compensate for the center of gravity position of the entire pendulum. Final calibration of the mercury column height is performed during the pendulum assembly process by adding mercury drop by drop! In order to have the same temperature at any moment as mercury has, which is an essential prerequisite for compensation, the pendulum rod is always immersed in it.

Although mercury performs good compensation for the pendulum temperature dilatation, its use is restricted by the fact that it is a toxic liquid that pollutes the environment it is discharged into, or evaporates into the atmosphere during accidents. That is the reason why other technical solutions are applied. John Harrison $^{5}$ was the first to use compensation based on steel and brass combination in building his stationary clocks and chronometer H1. It is the so-called gridiron bimetal pendulum, whose conceptual solution is given in Fig. 2. The pendulum support is built of 5 steel and 4 brass parallel and symmetrical rods. They are equal in length and are connected in such a way that steel rods always expand from and brass rods toward the suspension point.

2 Wiener Uhren - precision wall clocks were handmade in the Austro-Hungarian Empire throughout the $19^{\text {th }}$ Century; well-known for accuracy, reliability and beauty of style.

3 Solid solution of $36 \%$ nickel in iron (symbol - $64 \mathrm{FeNi}$ ); Swiss scientist Charles Edouard Guillaume discovered this alloy in 1896 and was awarded the Nobel Prize in Physics in recognition of his discovery of nickel-steel alloys.

4 Honorable George Graham (1673-1751) was one of the most famous English clockmakers who invented the deadbeat escapement. He was made Master of Worshipful Company of Clockmakers in 1722.

5 John Harrison was a great English clockmaker and inventor. He built the first marine chronometer, and solved the 'problem of longitude'. 


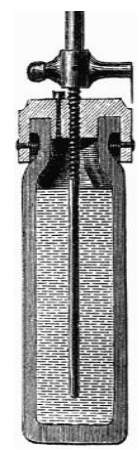

Figure 1

Glass vial of a mercury pendulum

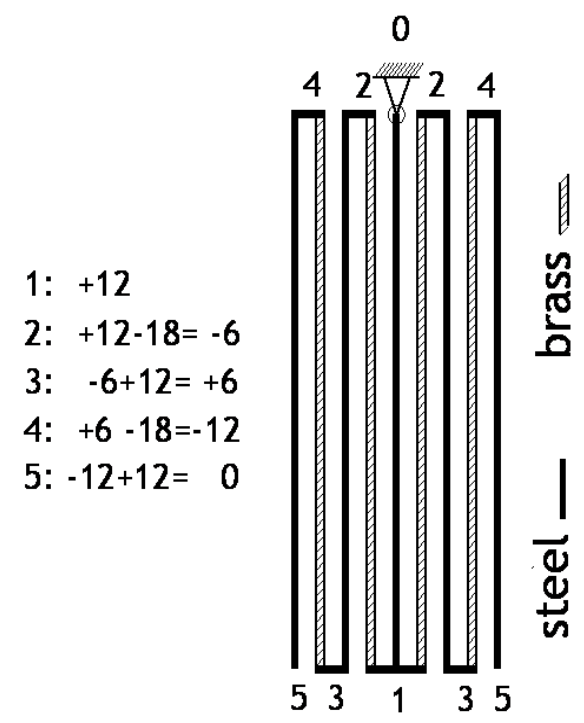

Figure 2

Bimetal gridiron pendulum

As linear coefficient of thermal dilatation for steel is $\mathbf{a}_{\mathrm{q}}=12 \cdot 10^{-6} \mathrm{~K}^{-1}$, and for brass $\mathbf{a}_{\mathrm{M}}=18 \cdot 10^{-6} \mathrm{~K}^{-1}$, the points $1,2,3,4$ relative to the gridiron pendulum suspension point have displacements $+12,-6,+6$ and $-12 \mu \mathrm{m} / \mathrm{Km}$, while the points $5-5$ are not subject to displacement due to temperature change. This solution, the practical realization being presented in Fig. 3, ensures thermal invariance of the position of the pendulum weight center of gravity. However, this is not enough as calculations will demonstrate below. The technical solution is known too. It is based on the same principle as the described gridiron pendulum but it uses steel and zinc coaxial pipes instead of steel and brass rods (Fig. 4). As zinc possesses an extremely high linear coefficient of thermal expansion, compensation is achieved only with one zinc and two steel pipes. 


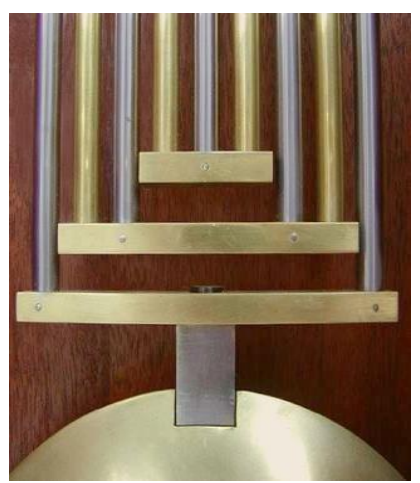

Figure 3

Technical realization of the gridiron pendulum

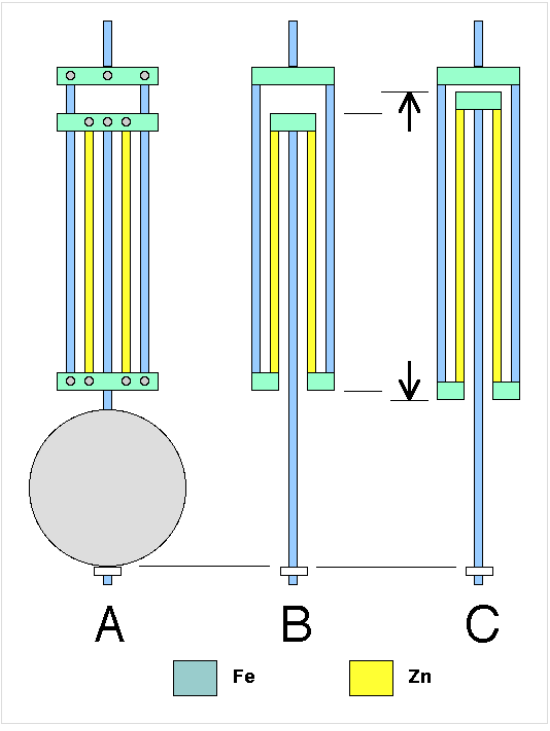

Figure 4

Cross-section of coaxial pipes of the pendulum steel and zinc rod

Very simple and, at the same time, almost the most efficient solution to the compensation for the pendulum thermal dilatation is accomplished by combining wood $^{6}$ and lead. If the pendulum rod is manufactured from the wood of silver fir and the weight from lead pipe of the corresponding length and mass, it is possible to achieve technically perfect temperature compensation for the reduced length of the entire pendulum. First, preliminary and then detailed calculations of such design is the subject of the analysis to follow.

6 Wood has first to be protected from harmful effects of moisture! 


\section{Preliminary Calculations of Thermal Compensation}

The wood of silver fir possesses extremely low, while lead high enough linear coefficient of thermal expansion $\left(a=4 \cdot 10^{-6} \mathrm{~K}^{-1}\right.$ and $\left.b=28 \cdot 10^{-6} \mathrm{~K}^{-1}\right)$, therefore their one-fold combination can completely compensate for the pendulum dilatation. Conceptual solution of this coupling is presented in Fig. 5. The pendulum support is a wood rod on the lower part of which a lead pipe is coaxially fixed. The rod expands thermally from the suspension point downward and a lead weight in opposite direction. Preliminary calculations are based on determining the ratio of the rod length to the pipe, thermal displacement of the center of gravity of the entire pendulum being thermally neutralized. From the fact that the position of the pendulum center of gravity is determined by it static moment $S$, where lengths depend on temperature, there follows:

$$
S=m \frac{l_{0}(1+a \theta)}{2}+M\left(l_{0}(1+a \theta)-\frac{L_{0}(1+b \theta)}{2}\right) ; \theta=t-t_{0}
$$

In this formula 6 as well as in the formulas 7 and 8 below $l_{0}$ and $L_{0}$ are the lengths of rod and lead pipe at the temperature $t_{0} ; m, M$ are rod and pipe masses; $a$ and b are linear coefficients of wood and lead thermal dilatation; $\theta=t-t_{0}$ is temperature difference.

To make the center of gravity position invariant relative to $\theta$, it is sufficient to annul the thermal gradient of the static moment:

$$
\frac{d S}{d \theta}=\frac{m \cdot l_{0} \cdot a}{2}+M \cdot l_{0} \cdot a-\frac{M \cdot L_{0} \cdot b}{2}=0
$$

As this gradient does not depend on the argument $\theta$, it follows that the influence of displacement position of the pendulum center of gravity will be completely neutralized if the following condition 8 is fulfilled:

$$
\lambda=\frac{l_{0}}{L_{0}}=\frac{b}{a} \cdot \frac{M}{2 M+m}
$$

The quality of this compensation will be checked using the example of the clock supplied with the described pendulum, for which it is adopted: $m=0.4 \mathrm{~kg}, M=20$ $\mathrm{kg}$ and $T=2 \mathrm{~s}$. For 10 days of permanent running, at temperature rise of $\theta=+10$ ${ }^{0} \mathrm{C}$, the clock is $-2.16 \mathrm{~s}$ late, which is 8 times lower compared to the thermally uncompensated equivalent $(-17.28 \mathrm{~s})$. The error residuum stems from the fact that the pendulum oscillation period is not only the function of the center of gravity position but also of the inertia square moment. Its reduction is the subject of the following, more complex calculations. 


\section{Optimal Solution of Thermal Compensation}

Fig. 5 illustrates the origin of error residuum in the timepiece running that has an installed pendulum with thermally compensated center of gravity: compared to the stationary pendulum center of gravity, by changing its original volume due to temperature change, a certain amount of material has changed the square moment of the system inertia. Better quality compensation has to include neutralization of this phenomenon too. Let us start from the formula 9 for a pendulum relative reduced length that is a function of temperature:

$$
\frac{l_{r}}{L_{0}}=\frac{\frac{1}{12} M(1+b \theta)^{2}+\frac{1}{3} m \lambda^{2}(1+a \theta)^{2}+M\left(\lambda(1+a \theta)-\frac{1}{2}(1+b \theta)\right)^{2}}{\frac{1}{2} m \lambda(1+a \theta)+M\left(\lambda(1+a \theta)-\frac{1}{2}(1+b \theta)\right)}
$$

The first, second and third term of the numerator in the above formula 9 originates from the square mass moments of inertia, in a row: eigenmoment of a lead pipe, total moment of a rod for the suspension point, and position moment of a lead pipe for the suspension point. The first and second term of the numerator originate from the static moments of inertia of the rod and lead pipe for the suspension point. In this analysis, we establish the condition of annulling the temperature gradient for the pendulum relative reduced length, described by formula 10:

$$
\begin{gathered}
\frac{1}{L_{0}} \cdot \frac{d l_{r}}{d \theta}=\frac{\frac{2}{3} a \lambda^{2} m(1+a \theta)+\frac{1}{6} b M(1+b \theta)-2\left(\frac{b}{2}-\lambda\right) M\left(\lambda(1+a \theta)-\frac{1}{2}(1+b \theta)\right)}{\frac{1}{2} \lambda m(1+a \theta)+M\left(\lambda(1+a \theta)+\frac{1}{2}(1+b \theta)\right)}- \\
-\frac{\left(\frac{a \lambda}{2}-\left(\frac{b}{2}-a \lambda\right) M\right) \cdot\left(\frac{1}{3} \lambda^{2} m(1+a \theta)^{2}+\frac{1}{12} M(1+b \theta)^{2}+M\left(\lambda(1+a \theta)-\frac{1}{2}(1+b \theta)\right)^{2}\right)}{\left(\frac{1}{2} \lambda(1+a \theta)+M\left(\lambda(1+a \theta)-\frac{1}{2}(1+b \theta)\right)\right)^{2}}
\end{gathered}
$$

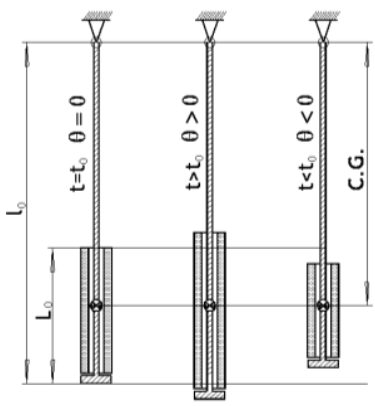

Figure 5

Temperature compensation for the pendulum center of gravity and origin of error residuum 
Table 2

Comparison of compensation quality

\begin{tabular}{|l||c||c||c||}
\hline \hline$\left.\Theta \Gamma^{0} \mathrm{C}\right]$ & $N K[\mathrm{~s}]$ & $K C G[s]$ & $K R L[s]$ \\
\hline \hline-30 & $+54,8416$ & $+6,48685$ & $-0,004220$ \\
\hline-20 & $+34,5607$ & $+4,32520$ & $-0,001876$ \\
\hline-10 & $+17,2802$ & $+2,16292$ & $-0,000469$ \\
\hline 0 & 0,0000 & 0,00000 & 0,000000 \\
\hline+10 & $-17,2798$ & $-2,16356$ & $-0,000469$ \\
\hline+20 & $-34,5593$ & $-4,32775$ & $-0,001876$ \\
\hline+30 & -51.8384 & $-6,49258$ & -0.004220 \\
\hline
\end{tabular}

From the fact that temperature gradient of the pendulum reduced length is a function of temperature, there follows an important statement: it is even theoretically impossible to achieve a complete compensation for the pendulum linear temperature dilatation! Yet, it is possible to have such a design solution that will reduce thermal disorders of the pendulum eigenoscillations to a minimum. In that regard, it would be the most appropriate to determine the value of the parameters $\lambda=l_{0} / L_{0}$ that will annul the gradient $d l_{r} / d \theta$ for the mean annual temperature of a place of timepiece running. The analysis was carried out using the example of a concrete timepiece supplied, as in a previous case, with a pendulum that the following parameters were adopted for: $m=0.4 \mathrm{~kg}, M=20 \mathrm{~kg}$ and $T=2 \mathrm{~s}$. Using the condition ${ }^{7}\left(d l_{r} l d \theta\right)=0$, and for $\theta=0{ }^{0} \mathrm{C}$, the parameter $\lambda=$ 3.0128 was determined, which leads to the pendulum total compensation at $\theta=0$ ${ }^{0} \mathrm{C}$. The lengths of a wood rod $l_{0}$ and a lead pipe $L_{0}$ are determined from the formula for the physical pendulum eigenoscillations period $\left(l_{0}=1178.815 \mathrm{~mm}, L_{0}\right.$ $=390.569 \mathrm{~mm})$. Table 2 shows error accumulation for three equivalent timepieces, during a 10-day running period, at 7 different temperatures. The first is supplied with an uncompensated pendulum (symbol - NK), the second has a pendulum with a compensated center of gravity (symbol - KCG) according to preliminary calculations, and the third has a pendulum with a compensated gradient of reduced length (symbol - KRL). The results tabulated indicate, first of all, the advantage of compensation for pendulum thermal dilatation, which is based on tempering the temperature gradient of reduced length, over neutralizing the center of gravity thermal displacement. In addition, it is evident that error residuum is always present, but in the last column it is almost negligible and more than acceptable for technical application.

7 From this condition there follows the cubic equation for $\lambda$ that has three real solutions: $\lambda 1=0.337667 ; \lambda 2=1.12793 ; \lambda 3=3.0128$; the last one is technically feasible. 


\section{Concluding Remarks}

The calculations of thermal gradient tempering of the pendulum relative reduced length, explained and carried out in this paper, can be also directly applied to combined Invar alloy (pendulum rod) and, for example, steel and brass (weight). The issue of non-uniform thermal conductivity, ever present in such technical solutions, is not of practical importance because changes in mean annual, and even daily, air temperatures are extremely slow. If calculations are performed without the help of any electronic aids, the problems of their numerical realization for $\left(d l_{r} l d \theta\right)=0$ are almost insurmountable. It could be the only reason why they were not performed in the $18^{\text {th }}$ and $19^{\text {th }}$ Century, but pendulum thermal compensation was done using tests, or by shortening the weight made of lead pipe. In this paper, all calculations were carried out in the Mathematics 5 application, fast and simply, with the aim to point to the strategy of numerical analysis itself that will make laboratory tests of metric adjustments, always long and expensive, shorter or even redundant.

\section{References}

[1] Popkonstantinović, B.,Miladinović, Lj., Stoimenov, M., Petrović, D., Petrović, N., Ostojić, G., Stankovski, S., The Practical Method for Thermal Compensation of Long-Period Compound Pendulum, Indian Journal of Pure \& Applied Physics, Vol. 49(10), October 2011, ISSN 0019-5596, pp. 657-664

[2] Popkonstantinović, B.,Miladinović, Lj., Stoimenov, M., Petrović, D., Ostojić, G., Stankovski, S., Design, Modelling and Motion Simulation of the Remontoire Mechanism, Transactions of Famena, XXXV-2, 2011, ISSN 1333-1124, pp. 79-93

[3] K. V. Kislov, Temperature Variations in Linear Dimensions of Elements of a Broadband Seismometer Perceived by the Latter as Ground Vibration, Seismic Instruments, Volume 46, Number 1, ISSN 1934-7871, Allerton Press, Inc. distributed exclusively by Springer Science+Business Media LLC, 2010, pp. 21-26

[4] Agatsuma K, Uchiyama T, Yamamoto K, Ohashi M, Kawamura S, Miyoki S, Miyakawa O, Telada S, Kuroda K, Direct Measurement of Thermal Fluctuation of High-Q Pendulum, Physical review letters, ISSN 0031-9007, 2010

[5] Jie Luo, Cheng-Gang Shao and Dian-Hong Wang, Thermal Noise Limit on the Period of a Torsion Pendulum, Classical and Quantum Gravity, Volume 26, ISSN 1361-6382, IOP Publishing, 2009

[6] Tiberiu Tudorache, Mihail Popescu, FEM Optimal Design of Wind Energy-based Heater, Acta Polytechnica Hungarica Vol. 6, No. 2, 2009, pp. 55-70 
[7] Fevzi Kentli, Hüseyin Çalik, Matlab-Simulink Modelling of 6/4 SRM with Static Data Produced Using Finite Element Method, Acta Polytechnica Hungarica, Vol. 8, No. 6, 2011, pp. 23-42

[8] A Cumming, A Heptonstall, R Kumar, W Cunningham, C Torrie, M Barton, K A Strain, J Hough and S Rowan, Finite Element Modelling of the Mechanical Loss of Silica Suspension Fibres for Advanced Gravitational Wave Detectors, Classical and Quantum Gravity, ISSN 0264-9381, IOP Publishing, 2009

[9] V. E. Dzhashitov and V. M. Pankratov, On the Possibility of Control of Interconnected Mechanical and Thermal Processes in Nonlinear Temperature - Perturbed Dynamic Systems, Journal of Computer and Systems Sciences International, ISSN 1555-6530, MAIK Nauka/Interperiodica distributed exclusively by Springer Science+Business Media LLC., 2009, pp. 481-488

[10] Jie Luo and Dian-Hong Wang, An Improved Correlation Method for Determining the Period of a Torsion Pendulum, ISSN 0034-6748, Review of Scientific Instruments, Vol. 79, 094705, 2008

[11] Woodward P., Woodward on Time (Group 5: Pendulum and Their Suspensions; 1. Compensation of Pendulums; 3. A bimetallic compensator), ISBN 0-95096216-3, Bill Taylor and British Horological Institute, UK, 2006

[12] Woodward P., My Own Right Time - An Exploration of Clockwork Design (2. Theory and practice; 13. Error correction), ISBN 978-0-19856522-2, Oxford University Press, New York, 2003

[13] Matthys R. J., Accurate Clock Pendulums, Oxford University Press, USA, 2004

[14] Andri M. Gretarsson, Gregory M. Harry, Steven D. Penn, Peter R. Saulson, William J. Startin, Sheila Rowan, Gianpietro Cagnoli and Jim Hough, Pendulum Mode Thermal Noise in Advanced Interferometers: A Comparison of Fused Silica Fibers and Ribbons in the Presence of Surface Loss, Physics Letters A, Volume 270, Issues 3-4, ISSN 0375-9601, ScienceDirect, Elsevier, 2000. pp. 108-114

[15] Peter R. Saulson, Thermal Noise in Mechanical Experiments, Physical Review D, ISSN 05562821, Volume 42, Issue 8, American Physical Society, 1990, pp. 2437-2445 